\title{
Oral Administration Recombinant Bifidobacterium-LTB (B Subunit of Heat-Labile Enterotoxin) Enhances the OVA (Ovalbumin)-Specific sIgA in Jejunal Mucosa of Sprague-Dawley (SD) Rat
}

\author{
Yong-Ping Ma*, Ya-Ning Hao, Wei Tang, Rong-Rong Wang, Fa-Ping Yi, You-Quan Bu, Lu-Yu Zhang, \\ Fang-Zhou Song
}

Key Laboratory of Biochemistry and Molecular Biology, The Molecular Medicine and Cancer Research Center, Chongqing Medical University, Chongqing, China.

Email: *ypma0909@yahoo.com.cn

Received June $19^{\text {th }}, 2012$; revised July $20^{\text {th }}, 2012$; accepted July $30^{\text {th }}, 2012$

\begin{abstract}
The LTB of enterotoxigenic Escherichia coli (ETEC) expressed in Bifidobacterium infantis (BI) has been testified as mucosal adjuvant with co-vaccination BI-CfaB (the major fimbrial subunit) together in vivo in our previous study. In order to investigate the mucosal adjuvant effect of BI-LTB to purified antigens, we oral vaccinated SD rats with recombinant BI-LTB plus OVA (rBI-LTB + OVA), and wild type BI plus OVA (wBI + OVA), OVA and PBS (Phosphate buffered saline) were vaccinated as controls, respectively. The OVA-specific sIgA in jejunal mucosa and specific IgG in serium were measured with ELISA (Enzyme-linked immunosorbent assay) and the sIgA producing cells were detected with immunohistochemistry technology (IHC) and Qwin image manipulation tools subsequently. The results shown rBI-LTB could stimulate SD rats produce high titer OVA-specific sIgA in rBI-LTB + OVA group and the OVA-specific sIgA titer in rBI-LTB + OVA group was found significant greater than that of the wBI + OVA group or OVA single group $(p<0.05)$. However no such significant difference was detected between the group wBI + OVA and OVA. IHC results suggested that intestinal mucosa and submucosa was the main field of sIgA secretion. These results suggested that recombinant LTB expression in BI could be used as a wide range mucosal adjuvant with different form antigens.
\end{abstract}

Keywords: Bifidobacterium infantis; LTB; OVA; Mucosal Adjuvant

\section{Introduction}

The LTB of Escherichia coli is one of bacterial products with the greatest potential to function as mucosal adjuvant. LTB belongs to ADP-ribosylating enterotoxins (cholera toxin and the heat-labile enterotoxin of E. coli). Both LTB and CTB (cholera toxin B subunit) recognize and bind to mucosal cell surface via their receptor GM1-ganglioside existed ubiquitously on the surface of mammalian cells [1-3]. The adjuvanticity of LTB has been directly related to GM1-binding activity and the interaction between LTB and the receptor activates B and $\mathrm{CD}^{+} \mathrm{T}^{-}$cells; and enhances antigen presentation by activating DCs (Dendritic cells) and other APCs (antigen presenting cells) through receptor-mediated endocytosis mechanisms [4]. Scientists explain that these molecules exert their adjuvant function by interacting with a variety

"Corresponding author. of cell types, including epithelial cells and DCs, etc. [5]. LTB activates selective differentiation of lymphocyte populations and increases presentation on MHC (Major histocompatibility complex) class II, which may be the basis for its adjuvant effect [6-8].

LTB and cholera toxin B (CTB) proteins have been successfully expressed in different expression systems, such as intracellular production in Bifidobacterium infantis (BI), Mycobacterium bovis and Lactobacillus or Bacillus brevis surface-displaced on Staphylococcus xylosus and $S$. carnosus, or as a secreted protein in a yeast expression system [9-15].

Lactic acid bacteria (LAB) have proved to be effective mucosal delivery vehicles that overcome the problem of delivering functional proteins to the mucosal tissues. BI, a special $\mathrm{LAB}$, is a Gram-positive, probiotic and anaerobic bacterium belonging to Bifidobacterium in human intestinal [16]. We testified BI is suitable to human oral 
vaccine development and the recombinant BI-LTB (rBI-LTB) co-vaccination with recombinant BI-CfaB of ETEC in vivo has mucosal adjuvant function in previous study [9]. However, we do not know whether rBI-LTB confers adjuvantity to purified antigens. The aim of the present study is to investigate the mucosal adjuvant effect of oral administration rBI-LTB on purified antigen OVA.

\section{Materials and Methods}

Bacterial strains and growth conditions. E. coli $\mathrm{DH} 5 \alpha$ strain was propagated in LB medium (Luria-Bertani) and used as host cell for molecular cloning; pBEX-LTB was constructed as described previously [9].

BI strain was cultured in MRS broth (Difco) containing $0.25 \%$ l-cysteine- $\mathrm{HCl}(\mathrm{w} / \mathrm{v}, \mathrm{pH} 7)$, at $37^{\circ} \mathrm{C}$ under anaerobic conditions. $50 \mu \mathrm{g} \cdot \mathrm{mL}^{-1}$ of ampicillin was added to both recombinant $\mathrm{BI}$ and $E$. coli strains when required.

Animals and Immunization. Sprague-Dawley (SD) rats of 3 - 4 weeks of age (male) were obtained from the experimental animal center (Chongqing Medical University, China) and divided into four groups as A, B, C and D. After treating with saturated sodium bicarbonate $(0.3$ $\mathrm{ml}$ each rat), the rats were intragastrically vaccinated three times on day 0,7 and 14 with rBI-LTB + OVA, wBI + OVA, OVA and PBS, The BI dose was $1.0 \times 10^{10}$ $\mathrm{CFU} \mathrm{mL} \mathrm{m}^{-1}$ and that of OVA was $10 \mu \mathrm{g}$ per rat on 3 occasions (Table 1).

Immunological assay (IMA). Blood samples were individually collected from immunized rats by a tail bleed on days $0,7,14$ and 21 for the analysis of systemic OVA-specific antibodies (10e10 bateria, on 3 occasions).

Fresh fecal pellets were individually collected lyophilized from the same rat groups on days $0,7,14$ and 21. The samples were treated as previous described [9] (Ma et al., 2011). The supernatants were analyzed for OVA-specific sIgA levels to study the secretory mucosal immune response. Both the serum was analyzed with goat anti-rat IgG and the fecal specific antibody titers were analyzed with goat anti-rat IgA by ELISA, respectively. OVA $\left(1.0 \mu \mathrm{g} \cdot \mathrm{mL}^{-1}\right)$ was diluted in a coating buffer $(50 \mathrm{mM}$ sodium carbonate, $30 \mathrm{mM}$ sodium bicarbonate, $\mathrm{pH}$ 9.6) and allowed to adsorb to 96-well plates

Table 1. The SD rats and the vaccination groups.

\begin{tabular}{ccc}
\hline Groups (rats) & Vaccinations agents & Dose ((C.F.U. $\left.\mathrm{mL}^{-1}\right)+\mu \mathrm{g}$ OVA $)$ \\
\hline $\mathrm{A}(\mathrm{n}=12)$ & rBI-LTB + OVA & $1.0 \times 10^{10}+10 \mu \mathrm{g}$ \\
$\mathrm{B}(\mathrm{n}=12)$ & wBI + OVA & $1.0 \times 10^{10}+10 \mu \mathrm{g}$ \\
$\mathrm{C}(\mathrm{n}=12)$ & OVA & $10 \mu \mathrm{g}$ \\
$\mathrm{D}(\mathrm{n}=10)$ & PBS & $/$ \\
\hline
\end{tabular}

$\left(1.0 \mu \mathrm{g}\right.$ each well) overnight at $4^{\circ} \mathrm{C}$. The plates were blocked with $5 \%$ skim milk for $1 \mathrm{~h}$ at room temperature and a twofold serial dilution of serum was added. After incubation, the plates were washed three times with PBS with $0.05 \%$ Tween 20 (PBS-Tween), and then 1.0 $\mathrm{mg} \cdot \mathrm{mL}^{-1}$ of goat anti-rat IgG mAb or anti-rat $\operatorname{sIgA} \mathrm{mAb}$ conjugated to HRP $\left(1.0 \mu \mathrm{g} \cdot \mathrm{mL}^{-1}\right.$, BOSTER, China) was added and incubated again.

The $\mathrm{OD}_{450}$ value was measured on a Molecular Devices SpectroMax Plus spectrophotometer. Endpoint titers were determined as the dilution of each sample showing a 2.1-fold higher level of absorbance at $450 \mathrm{~nm}$ than that of the negative control samples. Average $\mathrm{OD}_{450}$ values for the animals were calculated.

Tissue samples preparation. Following collection of blood and fecal samples, two of vaccinated rats were randomly anaesthetised and sacrificed from each group and the jejunum samples were isolated from each rat on days 7, 14 and 21, respectively. Following wash with PBS, the jejunum samples were fixed with $4 \%$ polyoxymethylene for $24 \mathrm{~h}$. Then the fixed jejunums were rinsed with $95 \%$ ethyl and successively dehydrated $10 \mathrm{~m}$ with ethyl from concentration $90 \%, 95 \%$ and $100 \%$, respectively. After treating with dimethylbenzene, the samples were embedded $4 \mathrm{~h}$ with paraffins at $56^{\circ} \mathrm{C}$ and cut into slices $(<5 \mu \mathrm{m})$. Then the slices were treated with acetone containing 1\% APES (3-aminopropyl triethoxysilane) and dried at $37^{\circ} \mathrm{C}$.

Immunohistochemical staining sections (IHCS). 1) The prepared slice samples were twice treated $40 \mathrm{~m}$ with dimethylbenzene to dewax and then successively dehydrated with ethyl from concentration $100 \%, 95 \%, 90 \%$, $80 \%$ to $70 \%$ for 30 s, respectively; 2) Following rinse 5 $\mathrm{m}$ with PBS three times, each slice was incubated $30 \mathrm{~m}$ with $50 \mu \mathrm{L}$ peroxidase blocking solution $(198 \mathrm{~mL}$ methanol $+2 \mathrm{~mL} 30 \%$ hydrogen peroxide) at $25^{\circ} \mathrm{C} ; 3$ ) After rinsing $5 \mathrm{~m}$ with distilled water, the slices were put into $0.01 \mathrm{M}$ citric acid solution $(\mathrm{pH} 6.0)$ and heat $15 \mathrm{~m}$ at $95^{\circ} \mathrm{C}$. Then, the slices were naturally cooled to $25^{\circ} \mathrm{C}$ and rinsed with PBS three times; 4$)$ Incubated with OVA (1.0 $\mu \mathrm{g} \cdot \mathrm{mL}^{-1}$ ) at $37^{\circ} \mathrm{C}$ for $3 \mathrm{~h}$ and then rinsed $5 \mathrm{~m}$ with PBS three times; 5) Following blocked with 5\% skim milk, each slice was incubated at $37^{\circ} \mathrm{C}$ for $30 \mathrm{~m}$ and then sucked up the serum with filter paper; 6) Incubated with OVA antibody (goat anti-OVA/HRP) at $37^{\circ} \mathrm{C}$ for $3 \mathrm{~h}$ and then rinsed $5 \mathrm{~m}$ with PBS three times; 7) Incubated $3 \mathrm{~m}$ with $100 \mu \mathrm{L}$ DBA solution and rinsed with tap water; 8) Following counterstain $3 \mathrm{~m}$ with hematine, then rinsed with tap water and overnight at $50^{\circ} \mathrm{C}$;9) Coverslip with neutral balsam.

ICH analysis (ICHA). All slices were surveyed and took pictures with Leica DM2000 microscope. Each slice randomly selected ten visual fields to statistical analysis 
by Qwin image manipulation tools. The yellowish-brown value stands for the positive cell area.

Statistical analysis. The data was statistically evaluated by the SPSS 19.0 statistical software package (SPSS Inc., Chicago, IL) and a value of $p<0.05$ was considered significant.

\section{Results}

IMA. Serum and mucosal OVA-specific antibody levels were measured by ELISA. High levels of serum OVAspecific IgG were observed on day 14 after the secondary booster vaccination, with an endpoint titer of 206 and reached a novel increased level in following booster dose on day 21 with the titer of 566 in groups $A(n=12)$. However, in group B $(n=12)$, the OVA-specific $\operatorname{IgG}$ titer just reached to 95 on $14 \mathrm{~d}$ and to 183 on $21 \mathrm{~d}$. In the other way, we did not find the OVA-specific IgG in group $C(n=12)$ and $D(n=10)$. Statistically, there was a significant difference in the $\operatorname{IgG}$ titer between the groups A and B for the adjuvant function of LTB ( $p<$ 0.05 ) (Figure 1). The level of variation in the responses was 20.48 in group A and 20.18 in group B between individual animals on day 21 .

Similar to the serum IgG titer variation, the fecal $\operatorname{sigA}$ titer in group A was greater than that in group B. High levels of OVA-specific sIgA was observed on day 14 after the secondary booster vaccination, with an endpoint titer of 7.57 and reached a novel increased level in following booster dose on day 21 with the titer of 54.67 in groups A $(n=12)$. However, in group $B(n=12)$, the OVA-specific sIgA titer just reached to 3.02 on $14 \mathrm{~d}$ and to 9.70 on $21 \mathrm{~d}$. However, we did not find the OVAspecific $\operatorname{sig} A$ in group $C(n=12)$ and $D(n=10)$. The

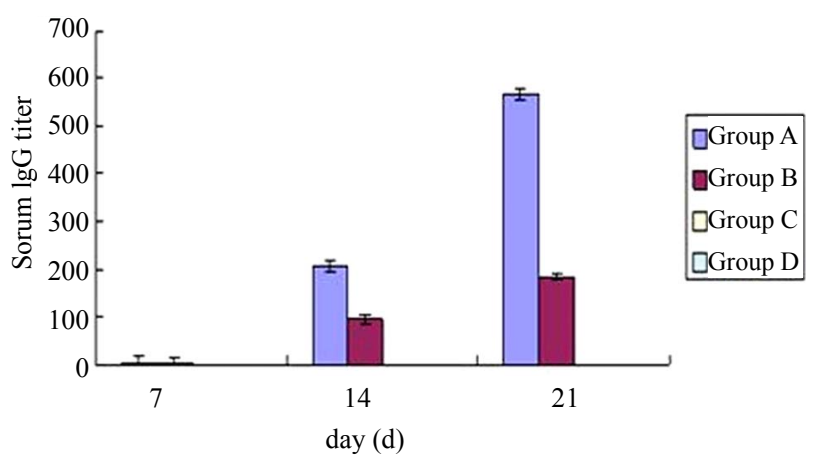

Figure 1. The serum IgG titer in different groups post-immunization. The group $A$ rats were immunized orally with rBI-LTB + OVA, group B with wBI + OVA, group C with OVA, and group D were treated with PBS (10e10 bateria, on 3 occasions). The group A shown significant difference, compared with group B $(p<0.05)$. However, there was no statistically significant difference IgG titer between the group $C$ and group $D$ for no detectable specific IgG. results suggest that rBI-LTB expressed in BI had mucosal adjuvant activity in group A. Statistically, there was a significant difference in the $\operatorname{sg} \mathrm{A}$ titer between the groups A and B for the adjuvant function of LTB $(p<$ 0.05) (Figure 2). The level of variation in the responses was 10.88 in group A and 0.61 in group B between individual animals on day 21 .

ICHA. Examined under a microscope, the OVA-specific SIgA producing cells were stained in yellowish-brown and distributed in jejunal mucosa, submucosa, external and internal of intestinal crypt, intestinal lamina propria and serous membrane (Figures 3 (a)-(h)). However, the colored positive cells in serous membrane were obviously fewer than that in other type cells. So the results suggest that intestinal mucosa and submucosa was the main field of sIgA secretion.

Analysis the specific sIgA producing cells on $7 \mathrm{~d}$ post-vaccination statistically, we found that the positive cells were increased appreciably group A than that in group B, but there was no significant difference each other. However, the positive cells were increased significantly from secondary and third booster in group A than that in group B $(p<0.05)$. As was expected, the positive cells did not find in group $\mathrm{C}$ and $\mathrm{D}$ (Figure 4).

\section{Discussion}

Appropriate mucosal adjuvant is essential for oral immunization to elicit immune response. Besides of the ADP-ribosylating enterotoxins (CT and LTB), the other two bacterial products, synthetic oligodeoxy-nucleotides containing unmethylated $\mathrm{CpG}$ dinucleotides (CpG ODN), and mono-phosphoryl lipid A (MPL), were used As mucosal adjuvants. Both MPL and $\mathrm{CpG}$ act through

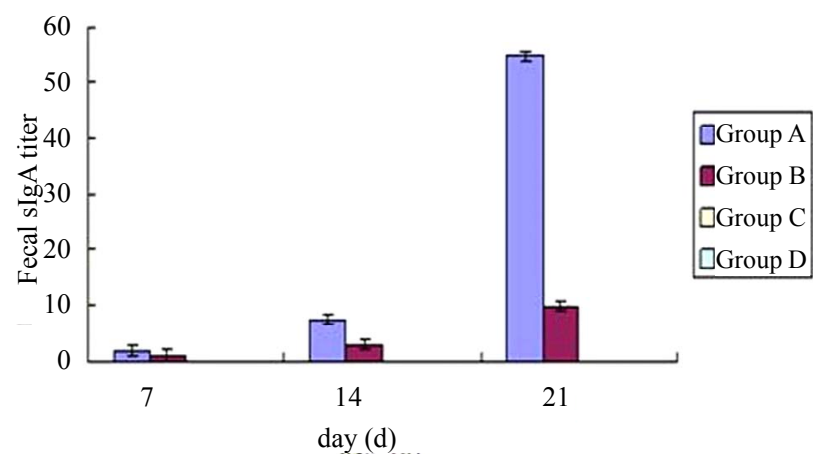

Figure 2. The fecal sIgA titer in different immunization groups in SD rat. The group A rats were immunized orally with rBI-LTB + OVA, group B with wBI + OVA, group C with OVA, and group D were treated with PBS (10e10 bateria, on 3 occasions). The group A shown significant difference, compared with group B $(p<0.05)$. However, there was no statistically significant difference sIgA titer between the group $\mathrm{C}$ and group $\mathrm{D}$ for no detectable specific sIgA. 


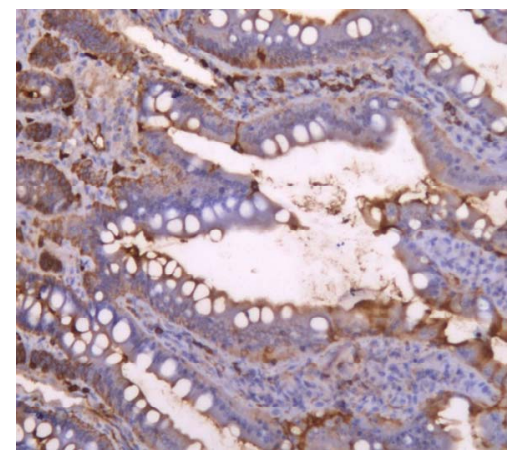

(a)

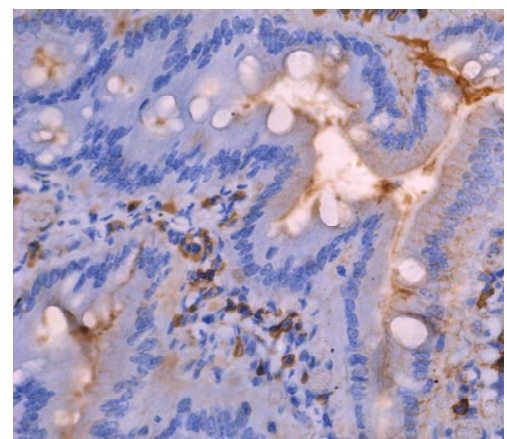

(c)

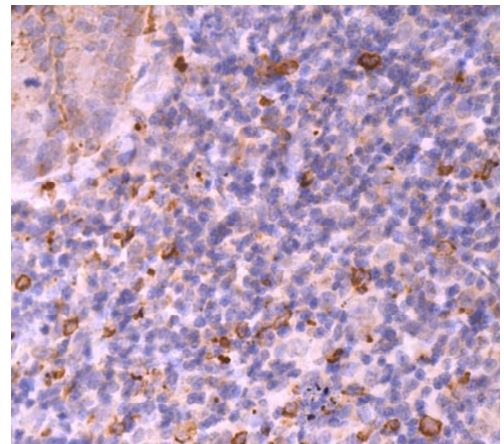

(e)

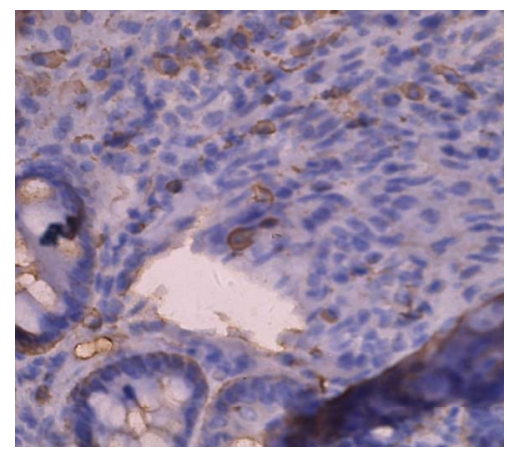

(g)

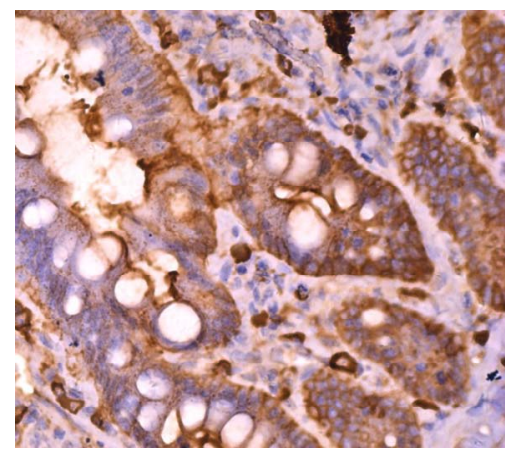

(b)

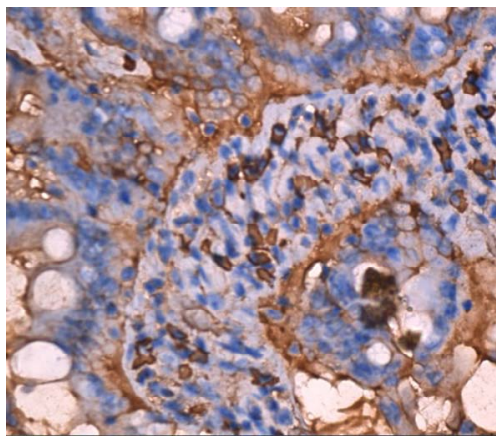

(d)

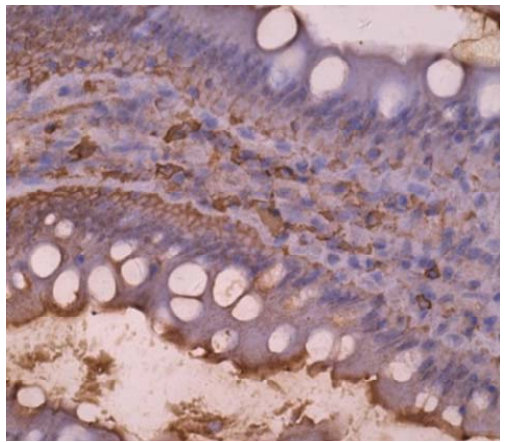

(f)

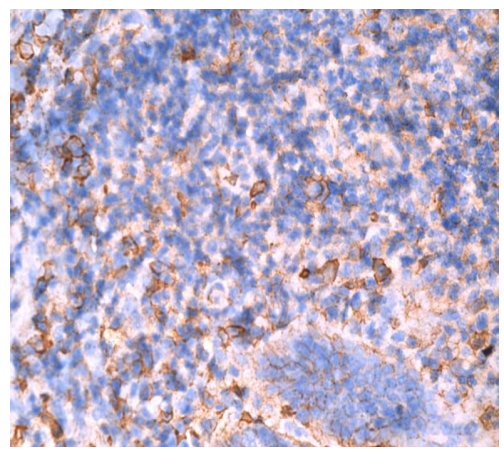

(h)

Figure 3. Immunohistochemical evaluation of OVA-specific producing cells in jenunal mucosa. (a), (b) Jenunal mucosal sections on $14 \mathrm{~d}$ and $21 \mathrm{~d}$ in group A (magnification 200 and 400, respectively). (c), (d) Sections on $14 \mathrm{~d}$ and $21 \mathrm{~d}$ in group B (magnification 200 and 400, respectively). (e), (f) Sections on $14 \mathrm{~d}$ and $21 \mathrm{~d}$ in group $\mathrm{C}$ (magnification 200 and 400 , respectively). (g), (h) sections on $14 \mathrm{~d}$ and $21 \mathrm{~d}$ in group $\mathrm{D}$ (magnification 400 and 200, respectively). The OVA-specific sIgA producing cells were stained with goat anti-OVA/HRP in yellowish-brown and distributed in jejunal mucosa, submucosa (arrows indicating mucosal locations of sIgA producing cells). 


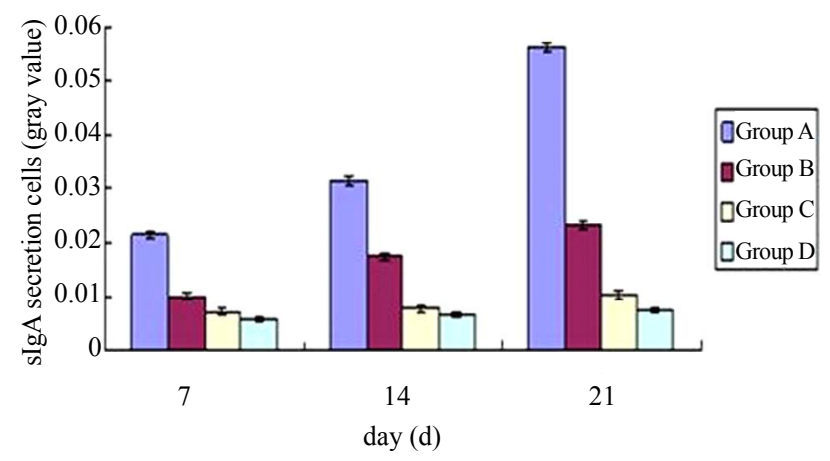

Figure 4. The specific sIgA secretion cells measured with yellow-brownish value. The positive cells, stained with goat anti-OVA/HRP, were increased significantly from secondary and third booster in group $A$ than that in group $B(p<0.05)$. However, the positive cells did not find in group $C$ and $D$.

MyD88-dependent and -independent pathways and the adjuvant activities of $\mathrm{CpG}$ and MPL are due to several different effects they have on innate and adaptive immune responses [5].

LTB is a nontoxic molecule with potent biological properties and is a powerful mucosal and parenteral adjuvant that induces a strong humoral and mucosal immune response against co-administered antigen or coupled antigens [8,9,15,17-19]. The adjuvant mechanism of LTB appears to be related with its capacity to: 1) enhance antigen presentation via MHC class I and MHC II; 2) activate selective differentiation of lymphocytes; 3 ) influence DCs maturation and activation; 4) induce B7-2 expression on APCs for subsequent co-stimulatory signaling to $\mathrm{CD} 4^{+}$T cells and; 5) increase the expression of activation markers on B lymphocytes (MHC class II, B7, CD40, CD25 and ICAM-1) [4,20].

Oral vaccination is based on antigen delivery to the gastrointestinal tract, the largest mucosal surface and the central site of $\operatorname{IgA}$ secretion [21]. Since the intestinal mucosa is the natural site of BI colonization, rLTB producing in BI easily crosses the epithelial layer to the area rich in cells of the mucosal immune system. BI-LTB appears to be one of the best forms of LTB adjuvant delivery system [9]. Because of the same reason, the several strains LAB has been genetically modified as a promising oral recombinant vaccine delivery system for inducing efficient mucosal immunity as well as systematic immunity [9,22-26].

Bifidobacterial cell wall preparation (whole peptidoglycan, WPG) has been documented with adjuvant activity and the activity of WPG is related to their ability to induce a reduction in regulatory $\mathrm{T}$ cells (Tregs) activity $[27,28]$. A previous document was demonstrated that LAB might be promising adjuvants in vaccines due to their capability to reduce functional activity of Tregs, thereby speeding up vaccine-induced immune responses
[29]. Another document suggests that WPG of bifidobacterium induces IL-12 secretion in DCs from bone marrow and rhIL-12, as adjuvant, has been shown to augment both cellular and humoral immunity [30-33]. In this study, we find that wild type BI performed weak adjuvant activity to OVA oral administration, which suggests that WPG of BI might be promising adjuvant activity in OVA.

There are many more experiments have been done in mice where there is more immunological reagents for these types of investigation. In this study, we use of the rat model is novel for oral vaccination. One reason for choosing rat is that a report used of rat for investigation of the role of intestinal bifidobacteria on immune system development [34]. They found that intestinal bifidobacteria plays an important role of development of both the gut and systemic immunity in early life. Neonatal SD rats supplemented daily with bifidobacteria could promote the maturation of DCs and its expression of IL-12 locally in the gut, favour the development of Th1 response by increasing the local and systemic expression of IFN $\gamma$ and ensure the intestinal Treg response by promoting the local expression of IL-10 [34].

There is no purified LT-B plus OVA as a comparable control to compare the organism expressing LT-B in this study. One reason is that the nontoxic CT plus OVA or LT-B plus OVA confirmed that the enterotoxin B subunit acts as a mucosal adjuvant intranasally immunized $[35,36]$. Oral vaccination is not an economically affordable way for purified LT-B or other enterotoxin B subunit because it needs high dose enterotoxin B subunit. However, BI expressing LT-B can easily get over it.

\section{Acknowledgements}

This study was funded by the National Natural Science Foundation of China (No. 30972585).

\section{REFERENCES}

[1] J. Sanchez and J. Holmgren, "Cholera Toxin-A Foe \& a Friend," Indian Journal of Medical Research, Vol. 133, No. 2, 2011, pp. 153-163.

[2] T. Yamamoto and T. Yokota, "Plasmids of Enterotoxigenic Escherichia coli H10407: Evidence for Two HeatStable Enterotoxin Genes and a Conjugal Transfer System," Journal of Bacteriology, Vol. 153, No. 3, 1983, pp. 1352-1360.

[3] T. Yamamoto, T. Tamura and T. Yokota, "Primary Structure of Heat-Labile Enterotoxin Produced by Escherichia coli Pathogenic for Humans," Journal of Biological Chemistry, Vol. 259, No. 8, 1984, pp. 5037-5044.

[4] V. P. da Hora, F. R. Conceição, O. A. Dellagostin and D. L. Doolan, "Non-Toxic Derivatives of LT as Potent Adjuvants," Vaccine, Vol. 29, No. 8, 2011, pp. 1538-1544. 
doi:10.1016/j.vaccine.2010.11.091

[5] L. C. Freytag and J. D. Clements, "Mucosal Adjuvants," Vaccine, Vol. 23, No. 15, 2005, pp. 1804-1813.

doi:10.1016/j.vaccine.2004.11.010

[6] N. A. Williams, "Immune Modulation by the CholeraLike Enterotoxin B-Subunits: From Adjuvant to Immunotherapeutic," Journal of Medical Microbiology, Vol. 290, No. 4-5, 2000, pp. 447-453. doi:10.1016/S1438-4221(00)80062-4

[7] T. O. Nashar, Z. E. Betteridge and R. N. Mitchell, "Evidence for a Role of Ganglioside GM1 in Antigen Presentation: Binding Enhances Presentation of Escherichia coli Enterotoxin B Subunit (EtxB) to CD4(+) T Cells," International Immunology, Vol. 13, No. 4, 2001, pp. 541-551. doi:10.1093/intimm/13.4.541

[8] E. Fingerut, B. Gutter, M. Goldway, D. Eliahoo and J. Pitcovski. "B Subunit of E. coli Enterotoxin as Adjuvant and Carrier in Oral and Skin Vaccination," Veterinary Immunology and Immunopathology, Vol. 112, No. 3-4, 2006, pp. 253-263. doi:10.1016/j.vetimm.2006.03.005

[9] Y. P. Ma, Y. L. Luo, X. P. Huang, F. Z. Song and G. L. Liu, "Construction of Bifidobacterium infantis as a Live Oral Vaccine that Expresses Antigens of the Major Fimbrial Subunit $(\mathrm{CfaB})$ and the B Subunit of Heat-Labile Enterotoxin (LTB) from Enterotoxigenic Escherichia coli," Microbiology, Vol. 158, No. 2, 2012, pp. 498-504.

[10] C. M. M. Hayward, P. O'Gaora, D. B. Young, G. E. Griffin, J. Thole, T. R. Hirst, L. R. R. Castello-Branco and D. J. M. Lewis. "Construction and Murine Immunogenicity of Recombinant Bacille Calmette Guerin Vaccine Expressing the B Subunit of Escherichia coli Heat Labile Enterotoxin," Vaccine, Vol. 17, No. 9-10, 1999, pp. 12721281. doi:10.1016/S0264-410X(98)00350-8

[11] N. Goto, J. I. Maeyama, Y. Yasuda, M. Isaka, K. Matano, S. Kozuka, T. Taniguchi, Y. Miura, K. Okhuma and K. Tochikubo, "Safety Evaluation of Recombinant Cholera Toxin B Subunit Produced by Bacillus brevis as a Mucosal Adjuvant," Vaccine, Vol. 18, No. 20, 2000, pp. 2164-2171. doi:10.1016/S0264-410X(99)00337-0

[12] M. Isaka, Y. Yasuda, S. Kozuka, T. Taniguchi, K. Matano, J. Maeyama, T. Komiya, K. Ohkuma, N. Goto and K. Tochikubo, "Induction of Systemic and Mucosal Antibody Responses in Mice Immunized Intranasally with Aluminium-Non-Adsorbed Diphtheria Toxoid Together with Recombinant Cholera Toxin B Subunit as an Adjuvant," Vaccine, Vol. 18, No. 7-8, 1999, pp. 743-751. doi:10.1016/S0264-410X(99)00258-3

[13] P. Slos, P. Dutot, J. Reymund, P. Kleinpeter, D. Prozzi, M. P. Kieny, J. Delcour, A. Mercenier and P. Hols, "Production of Cholera Toxin B Subunit in Lactobacillus," FEMS Microbiology Letters, Vol. 169, No. 1, 1998, pp. 29-36. doi:10.1111/j.1574-6968.1998.tb13295.x

[14] S. Liljeqvist, P. Samuelson, M. Hansson, T. N. Nguyen, H. Binz and S. Stahl, "Surface Display of the Cholera Toxin B Subunit on Staphylococcus xylosus and Staphylococcus carnosus," Applied and Environmental Microbiology, Vol. 63, No. 7, 1997, pp. 2481-2488.

[15] E. Fingerut, B. Gutter, R. Meir, D. Eliahoo and J. Pit- covski, "Vaccine and Adjuvant Activity of Recombinant Subunit B of E. coli Enterotoxin Produced in Yeast," Vaccine, Vol. 23, No. 38, 2005, pp. 4685-4696. doi:10.1016/j.vaccine.2005.03.050

[16] F. Guarner and J. R. Malagelada, "Gut Flora in Health and Disease," The Lancet, Vol. 361, No. 9356, 2003, pp. 512-519. doi:10.1016/S0140-6736(03)12489-0

[17] A. da Silva Ramos Rocha, F. R. Conceição, A. A. Grassmann, V. L. Lagranha and O. A. Dellagostin. "B Subunit of Escherichia coli Heat-Labile Enterotoxin as Adjuvant of Humoral Immune Response in Recombinant BCG Vaccination," Canadian Journal of Microbiology, Vol. 54, No. 8, 2008, pp. 677-686. doi:10.1139/W08-056

[18] F. R. Conceicao, A. N. Moreira and O. A. Dellagostin, "A Recombinant Chimera Composed of R1 Repeat Region of Mycoplasma hyopneumoniae P97 Adhesin with Escherichia coli Heat-Labile Enterotoxin B Subunit Elicits Immune Response in Mice," Vaccine, Vol. 24, No. 29-30, 2006, pp. 5734-5743. doi:10.1016/j.vaccine.2006.04.036

[19] R. Weltzin, B. Guy, W. D. Thomas Jr., P. J. Giannasca and T. P. Monath, "Parenteral Adjuvant Activities of EScherichia coli Heat-Labile Toxin and its B Subunit for Immunization of Mice against Gastric Helicobacter pylori Infection," Infection and Immunity, Vol. 68, No. 5, 2000, pp. 2775-2782. doi:10.1128/IAI.68.5.2775-2782.2000

[20] T. O. Nashar, T. R. Hirst and N. A. Williams, "Modulation of B-cell Activation by the B Subunit of Escherichia coli Enterotoxin: Receptor Interaction Up-Regulates MHC Class II, B7, CD40, CD25 and ICAM-1," Immunology, Vol. 91, No. 4, 1997, pp. 572-578. doi:10.1046/j.1365-2567.1997.00291.x

[21] J. R. McGhee, J. Mestecky, M. T. Dertzbaugh, J. H. Eldridge, M. Hirasawa and H. Kiyono, "The Mucosal Immune System: From Fundamental Concepts to Vaccine Development," Vaccine, Vol. 10, No. 2, 1992, pp. 75-88. doi:10.1016/0264-410X(92)90021-B

[22] J. K. Liu, X. L. Hou, C. H. Wei, L. Y. Yu, X. J. He, G. H. Wang, J. S. Lee and C. J. Kim, "Induction of Immune Responses in Mice after Oral Immunization with Recombinant Lactobacillus casei Strains Expressing Enterotoxigenic Escherichia coli F41 Fimbrial Protein," Applied and Environmental Microbiology, Vol. 75, No. 13, 2009 pp. 4491-4497. doi:10.1128/AEM.02672-08

[23] M. Medina, E. Vintiñi, J. Villena, R. Raya and S. Alvarez, "Lactococcus lactis as an Adjuvant and Delivery Vehicle of Antigens against Pneumococcal Respiratory Infections," Bioengineered Bugs, Vol. 1, No. 5, 2010, pp. 313-325. doi:10.4161/bbug.1.5.12086

[24] C. H. Wei, J. K. Liu, X. L. Hou, L. Y. Yu, J. S. Lee and C. J. Kim, "Immunogenicity and Protective Efficacy of Orally or Intranasally Administered Recombinant Lactobacillus casei Expressing ETEC K99," Vaccine, Vol. 28, No. 24, 2010, pp. 4113-4118. doi:10.1016/j.vaccine.2009.05.088

[25] J. Wells, "Mucosal Vaccination and Therapy with Genetically Modified Lactic Acid Bacteria," Annual Review of Food Science and Technology, Vol. 2, 2011, pp. 423445. doi:10.1146/annurev-food-022510-133640 
[26] S. Yamamoto, J. Wada, T. Katayama, T. Jikimoto, M. Nakamura, S. Kinoshita, K. M. Lee, M. Kawabata and T. Shirakawa, "Genetically Modified Bifidobacterium Displaying Salmonella-Antigen Protects Mice from Lethal Challenge of Salmonella Typhimurium in a Murine Typhoid Fever Model," Vaccine, Vol. 28, No. 41, 2010, pp. 6684-6691. doi:10.1016/j.vaccine.2010.08.007

[27] K. Sekine, E. Watanabe-Sekine, T. Toida, T. Kasashima, T. Kataoka and Y. Hashimoto, "Adjuvant Activity of the Cell Wall of Bifidobacterium infantis for in Vivo Immune Responses in Mice," Immunopharmacol Immunotoxicol, Vol. 16, No. 4, 1994, pp. 589-609. doi:10.3109/08923979409019741

[28] E. G. Schmidt, M. H. Claesson, S. S. Jensen, P. Ravn and N. N. Kristensen, "Antigen-Presenting Cells Exposed to Lactobacillus acidophilus NCFM, Bifidobacterium bifidum BI-98, and BI-504 Reduce Regulatory T Cell Activity," Inflammatory Bowel Diseases, Vol. 16, No. 3, 2010, pp. 390-400.

[29] D. Paineau, D. Carcano, G. Leyer, S. Darquy, M. A. Alyanakian, G. Simoneau, J. F. Bergmann, D. Brassart, F. Bornet and A. C. Ouwehand, "Effects of Seven Potential Probiotic Strains on Specific Immune Responses in Healthy Adults: A Double-Blind, Randomized, Controlled Trial," FEMS Immunology and Medical Microbiology, Vol. 53, No. 1, 2008, pp.107-113. doi:10.1111/j.1574-695X.2008.00413.x

[30] L. L. Zhao and Q. Cheng, "The Influence of Whole Peptidoglycan of Bifidobacterium on Interleukin-12 Produced by Dendritic Cells Derived from Bone Marrow of Mice in Vitro," Chinese Journal of Microecology, Vol. 21, No. 10, 2009, pp. 896-901.

[31] O. Hamid, J. C. Solomon, R. Scotland, M. Garcia, S. Sian, W. Ye, S. L. Groshen and J. S. Weber, "Alum with Interleukin-12 Augments Immunity to a Melanoma Peptide
Vaccine: Correlation with Time to Relapse in Patients with Resected High-Risk Disease," Clinical Cancer Research, Vol. 13, No. 1, 2007, pp. 215-222. doi:10.1158/1078-0432.CCR-06-1450

[32] M. A. Jacobson, E. Sinclair, B. Bredt, L. Agrillo, D. Black, C. L. Epling, A. Carvidi, T. Ho, R. Bains, V. Girling and S. P. Adler, "Safety and Immunogenicity of Towne Cytomegalovirus Vaccine with or without Adjuvant Recombinant Interleukin-12," Vaccine, Vol. 24, No. 25, 2006, pp. 5311-5319. doi:10.1016/j.vaccine.2006.04.017

[33] A. K. Wright, I. Christopoulou, S. El Batrawy, J. Limer and S. B. Gordon, "rhIL-12 as Adjuvant Augments Lung Cell Cytokine Responses to Pneumococcal Whole Cell Antigen," Immunobiology, Vol. 216, No. 10, 2011, pp. 1143-1147.

[34] P. Dong, Y. Yang and W. P. Wang, "The Role of Intestinal Bifidobacteria on Immune System Development in Young Rats," Early Human Development, Vol. 86, No. 1, 2010, pp. 51-58. doi:10.1016/j.earlhumdev.2010.01.002

[35] P. N. Boyaka, M. Ohmura, K. Fujihashi, T. Koga, M. Yamamoto, M. N. Kweon, Y. Takeda, R. J. Jackson, H. Kiyono, Y. Yuki and J. R. McGhee, "Chimeras of Labile Toxin One and Cholera Toxin Retain Mucosal Adjuvanticity and Direct Th Cell Subsets via Their B Subunit," Journal of Immunology, Vol. 170, No. 1, 2003, pp. 454462.

[36] S. Yamamoto, H. Kiyono, M. Yamamoto, K. Imaoka, K. Fujihashi, F. W. Van Ginkel, M. Noda, Y. Takeda and J. R. McGhee, "A Nontoxic Mutant of Cholera Toxin Elicits Th2-Type Responses for Enhanced Mucosal Immunity," Proceedings of the National Academy of Sciences USA, Vol. 94, No. 10, 1997, pp. 5267-5272. doi:10.1073/pnas.94.10.5267 